\section{FLNC missense variants in familial noncompaction cardiomyopathy}

Jaap I. van Waning, ${ }^{1}$

Yvonne M. Hoedemaekers, ${ }^{2}$

Wouter P. te Rijdt,, ${ }^{2,3}$ Arne I. Jpma, ${ }^{4}$

Daphne Heijsman, ${ }^{4}$ Kadir Caliskan, ${ }^{5}$

Elke S. Hoendermis, ${ }^{6}$

Tineke P. Willems, ${ }^{7}$

Arthur van den Wijngaard, ${ }^{8}$

Albert Suurmeijer,

Marjon A. van Slegtenhorst,

Jan D.H. Jongbloed,

Danielle F. Majoor-Krakauer, ${ }^{1}$

Paul A. van der $\mathbf{Z w a a g}^{2}$

${ }^{1}$ Department of Clinical Genetics, Erasmus Medical Center, Rotterdam; ${ }^{2}$ Department of Genetics, University of Groningen, University Medical Center Groningen, Groningen; ${ }^{3}$ Department of Pathology, University of Groningen, University Medical Center Groningen, Groningen; ${ }^{4}$ Department of Pathology, Erasmus Medical Center, Rotterdam; ${ }^{5}$ Department of Cardiology, Erasmus Medical Center, Rotterdam; ${ }^{6}$ Department of Cardiology, University of Groningen, University Medical Center Groningen, Groningen; ${ }^{7}$ Department of Radiology, University of Groningen, University Medical Center Groningen, Groningen; ${ }^{8}$ Department of Clinical Genetics, Maastricht University Medical Center, Maastricht, the Netherlands

\begin{abstract}
The majority of familial noncompaction cardiomyopathy (NCCM) is explained by pathogenic variants in the same sarcomeric genes that are associated with hypertrophic (HCM) and dilated (DCM) cardiomyopathy. Pathogenic variants in the filamin $\mathrm{C}$ gene (FLNC) have been linked to HCM and DCM. We expand the spectrum of FLNC related cardiomyopathies by presenting two families with likely pathogenic FLNC variants showing familial segregation of $\mathrm{NCCM}$ and concurrent coarctation of the aorta and/or mitral valve abnormalities.
\end{abstract}

\section{Introduction}

Noncompaction cardiomyopathy (NCCM) is characterized by excessive trabeculation of the left ventricle (LV) with a noncompacted to compacted ratio of more than 2 according to current echocardiographic criteria, or 2.3 on CMR. ${ }^{1,2}$ Approximately $10 \%$ of patients diagnosed with NCCM have concurrent congenital heart defects (CHD). ${ }^{3,4}$

In $30-40 \%$ of cases diagnosed with NCCM a pathogenic variant can be identified. Around $80 \%$ of these pathogenic variants involve the same sarcomere genes, that are the major causes for hypertrophic cardiomyopathy (HCM) and dilated cardiomyopathy (DCM), in particular $M Y H 7$, MYBPC3 and TTN. 5,6 Filamin C (FLNC) plays a central role in muscle functioning by maintaining the structural integrity of the muscle fibers. Pathogenic variants in FLNC were found to be associated with a wide spectrum of myopathies ranging from cardiomyopathies to distal skeletal myopathies. Truncating FLNC variants were previously associated with dilated cardiomyopathy $^{7-9}$ and missense variants were identified in familial HCM and restrictive cardiomyopathy. FLNC has not been associated with NCCM or CHD before.

We present two Dutch families where familial NCCM with CHD were linked to rare $F L N C$ missense variants. These observations suggest that the spectrum of clinical manifestations of $F L N C$ variants include familial NCCM with CHD

\section{Case Reports}

\section{Family A}

In this family (Figure 1A), a 52-yearold woman (II:3) was first diagnosed with NCCM when she underwent cardiologic examination for a suspected perimyocarditis. Echocardiography showed pericardial effusion and normal LV dimensions without LV dysfunction. The LV walls showed hypertrabeculation with end-systolic noncompacted/compacted $(\mathrm{NC} / \mathrm{C})$ ratio $>2$. Electrocardiographically, inferolateral repolarization abnormalities were observed. Cardiac magnetic resonance imaging (CMR) confirmed the diagnosis of NCCM with diastolic $\mathrm{NC} / \mathrm{C}$ ratio $>2.3$ in the $\mathrm{LV}$ inferoseptal wall. She had elevated CK levels of $1234 \mathrm{U} / \mathrm{L}$ [ref $<200 \mathrm{U} / 1]$. No signs for neuromuscular disease were detected at neurologic examination. After seven years of follow-up, she remained cardiologically asymptomatic (NYHA class I).

Family screening revealed NCCM in two relatives. A niece (III:3), was diagnosed with NCCM at age 21 and had surgery at age seven for coarctation of the aorta (CoA). She fulfilled both the echocardiography and $\mathrm{CMR}$ diagnostic criteria for NCCM and had excessive long chordae of
Correspondence: Jaap I. van Waning, Department of Clinical Genetics, EE 2038, Erasmus MC, POB 2040, 3000CA Rotterdam, the Netherlands.

Tel.: +3107038388 - Fax: +3107043072.

E-mail: j.vanwaning@erasmusmc.nl

Acknowledgements: JVW was supported by a grant from the Jaap Schouten Foundation. WPTR was supported by a Young Talent Program (CVON PREDICT) grant 2017T001 - Dutch Heart Foundation.

Conflict of interest: the authors declare no potential conflict of interest.

Received for publication: 20 March 2019

Revision received: 29 July 2019.

Accepted for publication: 17 September 2019

This work is licensed under a Creative Commons Attribution NonCommercial 4.0 License (CC BY-NC 4.0).

(C) Copyright: the Author(s), 2019

Licensee PAGEPress, Italy

Cardiogenetics 2019; 9:8181

doi:10.4081/cardiogenetics.2019.8181

the anterior mitral valve leaflet (Figure 2A and $\mathrm{B}$ ). The noncompaction had not been recognized in the past on echocardiography. Cardiologic screening of an asymptomatic brother (II:4) at age 54 years showed that he also had NCCM; with a NC/C ratio of 2.3 on echocardiography and a ratio of 2.9 on CMR. No previous cardiac imaging had been performed. He had elevated CK-levels of $265 \mathrm{U} / \mathrm{L}$ without neuromuscular signs. Ten years after the diagnosis NCCM he had an episode of atrial fibrillation that required electric cardioversion. CMR from the brother (II:1) and the son (III:1) of the proband were performed at age 57 and 15 years, respectively, showing borderline $\mathrm{NC} / \mathrm{C}$ ratios of respectively 2.1 and 2.2 on MRI, i.e. just below the diagnostic criteria. Proband III-2 did not participate in the family screening.

\section{Family B}

In family $\mathrm{B}$ (Figure 1B) the diagnosis NCCM in a 17-year-old boy (III:1) was made by echocardiography. He was referred because of multiple unexplained episodes of syncope. He also had a ventricular septal defect (VSD) and a mild mitral valve prolapse (MVP). CMR revealed partial LV noncompaction from the apex to midventricular region with an $\mathrm{NC} / \mathrm{C}$ ratio of 3.0 (Figure 2C and D). An implantable cardioverter-defibrillator (ICD) was implanted. After 9 years of follow-up, the LV function remained normal without ICD shocks. CK- 
levels were elevated (419-1188 U/L) in the absence of neuromuscular signs. His mother (II:2) was under cardiologic surveillance because she had a VSD, MVP, CoA and a bicuspid aortic valve. The CMR showed that she complied for the diagnostic criteria for NCCM with a NC/C ratio of 2.4. She had diastolic LV dysfunction, with preserved LV systolic function and underwent multiple cardiac ablations for atrial fibrillation. At age 44 years she experienced severe bradycardia, which necessitated cardiac resuscitation, resulting from combined flecainide and metoprolol treatment. A pacemaker was implanted. Her highest CK-level was 1174 U/L. The proband's brother (III:2) also suffered multiple episodes of syncope and was diagnosed with NCCM at age 19, with a NC/C ratio of 3.1 on CMR. His highest CK-level was 294U/1. Proband III-3 was screened cardiologically and had no signs of NCCM on echocardiography. No DNA analysis was performed.

\section{Genetic testing}

Diagnostic DNA NGS targeted testing of a panel of 54 cardiomyopathy genes, that did not include FLNC, as presented in Table 1 , did not reveal a genetic causes for NCCM in the two index cases. Also singlenucleotide polymorphism-array DNA testing showed no structural DNA changes. Subsequently, whole exome sequencing was performed in the NCCM patients II:1, III:1 and III:3 from family A and III:1, III:2, and II:2 from family B. Patients II-2 from family B was included because we suspected that a causative mutation may underlie a spectrum of cardiac phenotypes. Written informed consent was obtained from all participating family members. The investigation conforms to the principles outlined in the Declaration of Helsinki. Variants were annotated using ANNOVAR ${ }^{10}$ and filtered using an in-house developed pipeline. Only variants segregating within each family, affecting exons or splice sites, with a population frequency below 0.01 in ExAC, NFE, GoNL were kept. For in silico prediction of the effect of nonsynonymous variants we used align GVGD, ${ }^{11}$ SIFT $^{12}$ and Polyphen ${ }^{13}$ and ensemble scores LR and Radial SVM. ${ }^{14}$ We selected variants who were predicted to be damaging by 3 of 5 prediction programs.
Segregating synonymous variants and variants predicted to be tolerated were excluded. In Family A, two candidate genes remained after filtering, MYH4 and FLNC, of which only the last was previously associated with cardiomyopathy. A variant in FLNC (c.6397C $>$ T, p.(Arg2133Cys), NM_001458.4, confirmed by sanger sequencing) segregated with the cardiac phenotype of NCCM in the three NCCM patients and in the two relatives with borderline NCCM features. A variant in the same location (p.(Arg2133His)) was previously reported in a family with HCM and classified as probably pathogenic. ${ }^{15}$ In family B, a novel $F L N C$ variant (c. $7177 \mathrm{C}>\mathrm{T}$, p.(Pro2393Ser)) was identified. The two FLNC variants were absent in the Genome Aggregation Database (http://gnomad. broadinstitute.org), affect highly conserved amino acids, and were predicted to be deleterious by multiple in silico prediction programs. No FLNC variants were found in thirteen unrelated NCCM patients without a CHD and without a pathogenic variant in 48 cardiomyopathy genes.

\section{Histology}

Right ventricular endomyocardial biopsy (RVEMB) samples from the proband of
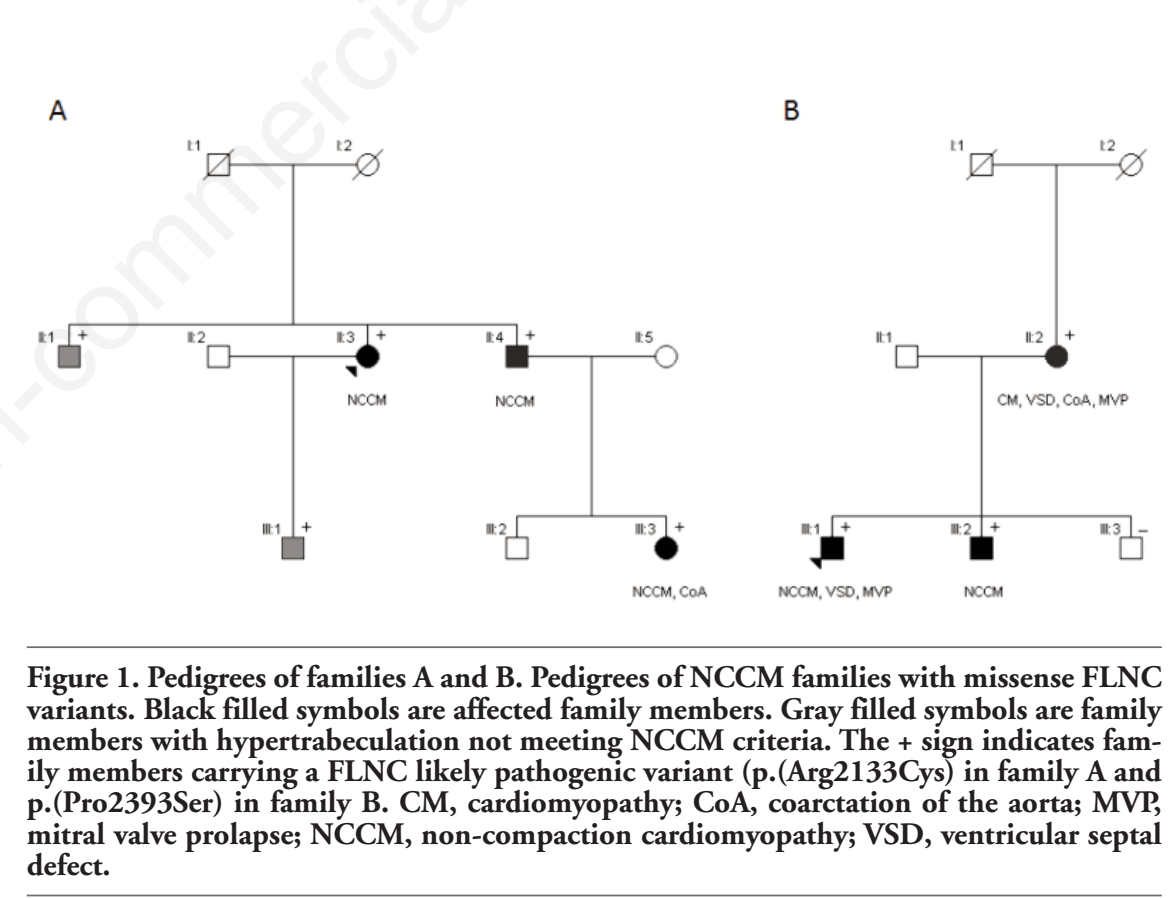

Figure 1. Pedigrees of families A and B. Pedigrees of NCCM families with missense FLNC variants. Black filled symbols are affected family members. Gray filled symbols are family members with hypertrabeculation not meeting NCCM criteria. The + sign indicates family members carrying a FLNC likely pathogenic variant (p.(Arg2133Cys) in family A and p.(Pro2393Ser) in family B. CM, cardiomyopathy; CoA, coarctation of the aorta; MVP, mitral valve prolapse; NCCM, non-compaction cardiomyopathy; VSD, ventricular septal defect. family B (III:1) were stained with hematoxylin and eosin as well as Masson's trichrome. To visualize protein aggregation and autophagic activity in cardiomyocytes, immunohistochemistry for microtubuleassociated protein 1A/1B-light chain 3 (LC3) was performed, as described previously. ${ }^{16}$ Light microscopic analysis showed nonspecific cardiomyopathic changes of myocyte hypertrophy and increased interstitial fibrosis (Figure 3A). The RV did not show an excessively thickened endocardial layer or hypertrabeculation, or intracellular aggregates or autophagic activity (Figure 3B).

\section{Discussion and conclusions}

This is the first report linking FLNC to NCCM in two families with rare FLNC variants. In these two families the cardiac phenotype included NCCM, NCCM with concurrent $\mathrm{CoA}$, NCCM with concurrent with a complex CHD consisting of VSD, $\mathrm{P}, \mathrm{CoA}$ and bicuspid aortic valve, and suggest that missense FLNC variants may VSD and MPV and also a NCCM patient

Table 1. Diagnostic DNA NGS targeted testing of a panel of 54 cardiomyopathy genes (not including FLNC). 
cause familial NCCM with or without one or more structural heart defects. Missense FLNC variants in the $\mathrm{N}$ and $\mathrm{C}$ terminal domains of $F L N C$ have been associated with hypertrophic and restrictive cardiomyopathy, causing sarcomeric aggregates containing FLNC leading to sarcomere dysfunction. ${ }^{15}$ Other $F L N C$ domains were associated with myofibrillar myopathy, showing similar intracellular aggregates in skeletal muscles. ${ }^{17,18}$ A large study of inherited cardiovascular disease patients showed that truncating FLNC variants were associated predominantly with an overlapping phenotype of severe dilated- and arrhythmogenic cardiomyopathies. ${ }^{8}$ The cardiomyopathy phenotypes associated with $F L N C$ variants have not included NCCM so far, however signs of LV hypertrabeculation not fulfilling NCCM diagnosis in some carriers of a truncating $F L N C$ variants have been reported. ${ }^{8}$ FLNC has not been linked to CHD, sofar, to the best of our knowledge.

Aortic coarctation in NCCM patients seems rare with an estimated prevalence of less than $1 \% .{ }^{4}$ We report two families with familial NCCM and a likely pathogenic FLNC variant in which CoA occurred; in one family one patient had NCCM with concomitant CoA. In the other family a NCCM patient with the familial FLNC variant had complex congenital cardiac defects including a CoA. It remains to be elucidated how FLNC and other genes can cause a cardiomyopathy with or without a CHD, and skeletal myopathy in other patients. It may be that FLNC resembles MYH7 in that aspect. Because among all the known genes associated with cardiomyopathies as well as skeletal myopathies, $M Y H 7$ variants occurs the most frequent in NCCM, and is also linked to Ebstein anomaly with and without NCCM, HCM, DCM, or Laing distal myopathy. ${ }^{19,20}$ Suggesting that variants affecting distinct domains of sarcomeric proteins may define the spectrum of cardiac and skeletal muscle phenotypes.

Pathogenic $F L N C$ variants are expected to disrupt the structure of the sarcomeric protein, leading to the formation of protein aggregates resulting in an impairment of the sarcomere function. ${ }^{15,21}$ One of the identified variants in this study, FLNC p.(Arg2133Cys), may have a similar effect as the reported FLNC variant p.(Arg2133His) at the same location with another amino-acid substitution, that was shown to have disrupting actin aggregates in cardiac tissue. ${ }^{15}$ We found elevated CK levels in two of the three NCCM patients in both affected families. Elevated CK levels were also noted in a previous study regarding FLNC variants with myopathy but also in patients with only a cardiac phenotype of

HCM. ${ }^{15}$ For the novel FLNC variant p.(Pro2393Ser), we observed fibrosis in the RV myocardium samples, indicating a damaging effect of the variant on the cardiac muscle. Fibrosis was also observed in previous reports with FLNC mutations. ${ }^{8,15}$ The cardiac fibrosis observed in the patients with the $F L N C$ variant suggests that similar pro-fibrotic mechanisms may be involved as observed in MYBPC3 cardiomyopathy. ${ }^{22}$ Similarly, to the original report no signs of intracellular aggregates or autophagic activity in the RV of the patient or in patients with a FLNC related cardiomyopathy were noted. ${ }^{8,15}$ The RV of this patient did not show evidence for hypertrabeculation morphologically or on imaging. However this

A

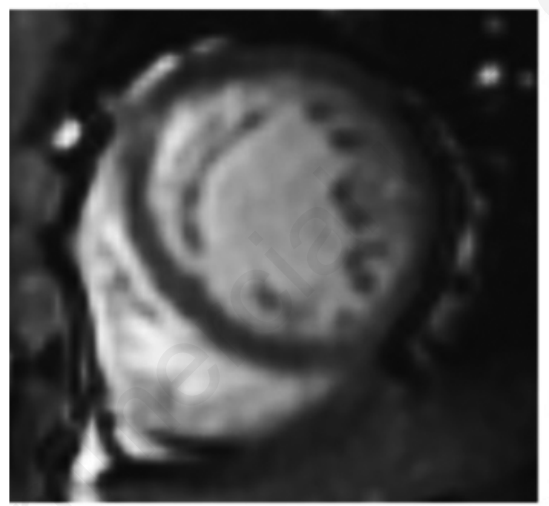

C
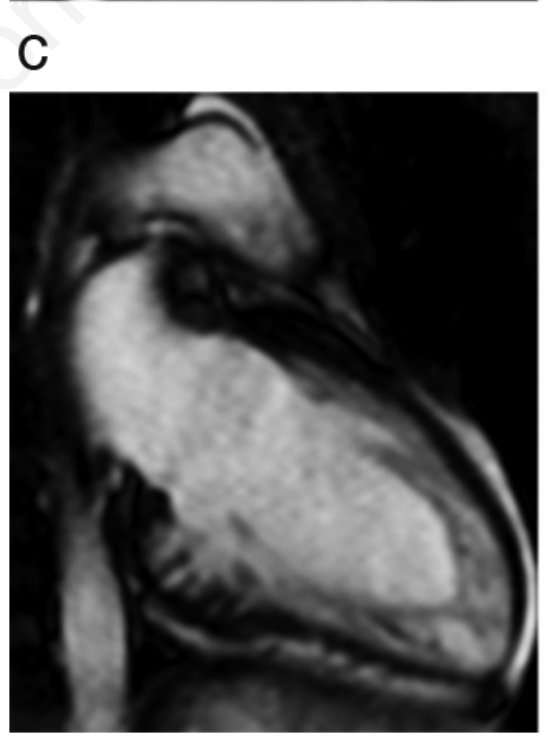

does not exclude an effect of the FLNC variant on the left ventricle, since RV hypertrabeculation in NCCM is rarely reported, and the NCCM presents predominantly with a LV phenotype.

As previous studies showed, genetics plays an important role in approximately half of the NCCM cases..$^{23}$ The genetics of NCCM are complex and affect mostly genes associated with myopathies including sarcomere or mitochondrial dysfunctioning. In this perspective FLNC fits into the genetically heterogeneous background of NCCM. Further studies are needed to assess the exact role and mechanisms of FLNC in NCCM, aortic coarctation and mitral valve abnormalities.
Figure 2. Imaging of two NCCM patients. Family A. patient III.3; A) Short axis of the left ventricle on cardiac magnetic resonance (CMR) showing the prominent trabeculae and intertrabecular recesses in NCCM; B) Echocardiogram of the LV of patient (III.3) with NCCM and excessive long chordae of the anterior mitral valve leaflet (arrow). Family B. patient III.1; C) Two-chamber long-axis of the left ventricle on CMR; D) Short axis view of the left ventricle on CMR showing the prominent trabeculae and intertrabecular recesses in NCCM. 


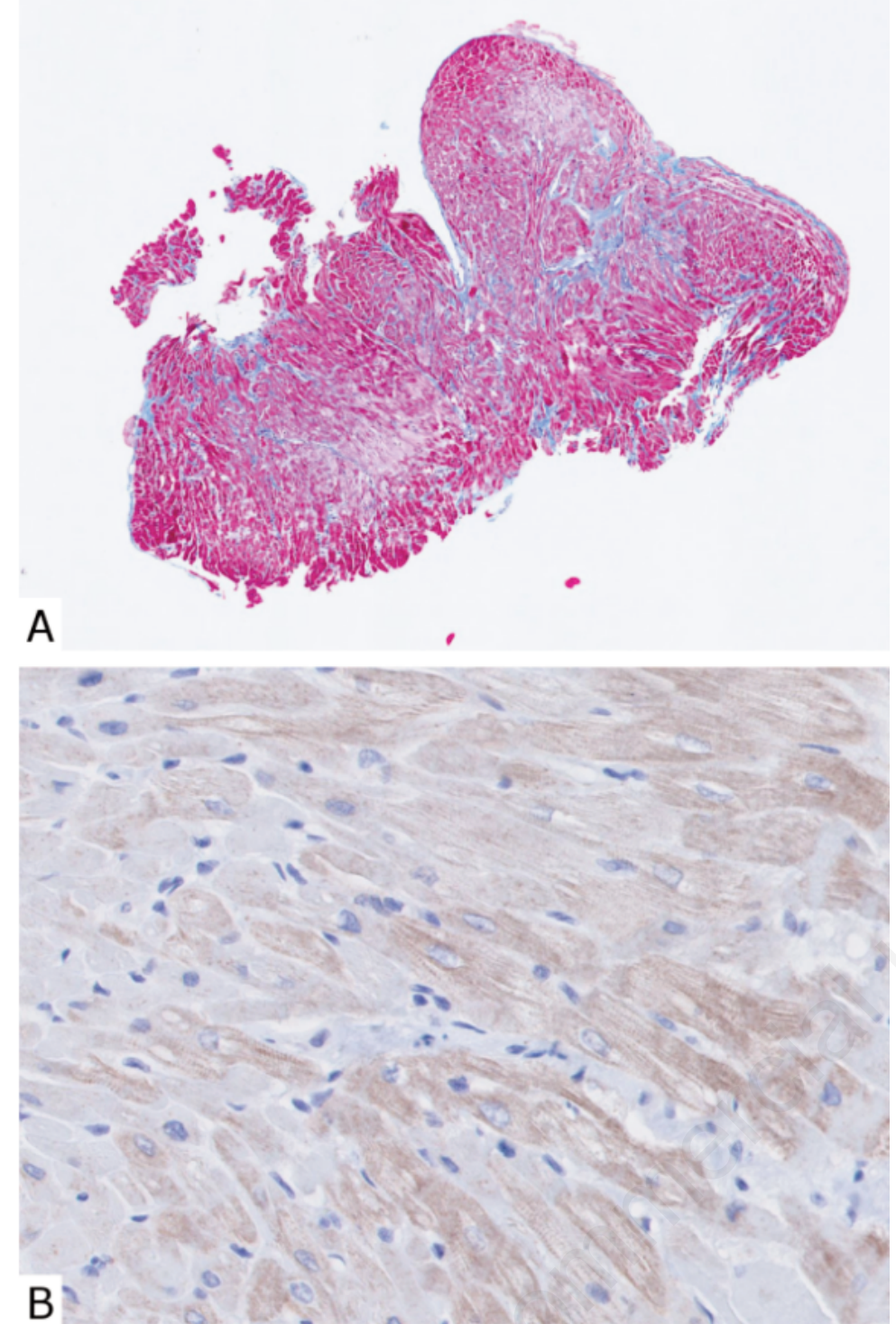

Figure 3. Myocardial tissue staining of FLNC likely pathogenic variant carrier (Family B. III:1). Myocardial tissue analysis of RVEMB samples from FLNC likely pathogenic variant carrier (Family B. III:1). A) Masson's trichrome staining, original magnification x20, shows mild fibrosis; B) Immunohistochemical staining (LC3), original magnification $\mathrm{x} 200$, does not show protein aggregates with autophagic activity in cardiomyocytes. Faint LC3 background cytoplasmic staining can be observed.

\section{References}

1. Chin TK, Perloff JK, Williams RG, et al. Isolated noncompaction of left ventricular myocardium. A study of eight cases. Circulation 1990;82:507-13.

2. Petersen SE, Selvanayagam JB, Wiesmann F, et al. Left ventricular noncompaction: insights from cardiovascular magnetic resonance imaging. J Am Coll Cardiol 2005;46:101-5.

3. Towbin JA, Lorts A, Jefferies JL. Left ventricular non-compaction cardiomyopathy. Lancet 2015;386:813-25.

4. Stahli BE, Gebhard C, Biaggi P, et al. Left ventricular non-compaction: prevalence in congenital heart disease. Int J Cardiol 2013;167:2477-81

5. Hoedemaekers YM, Caliskan K, Michels M, Fet al. The importance of genetic counseling, DNA diagnostics, and cardiologic family screening in left ventricular noncompaction cardiomyopathy. Circ Cardiovasc Genet 2010;3:232-9.

6. Miszalski-Jamka K, Jefferies JL, Mazur $\mathrm{W}$, et al. Novel genetic triggers and genotype-phenotype correlations in patients with left ventricular noncompaction. Circ Cardiovasc Genet 2017;10:4.

7. Janin A, N'Guyen K, Habib G, et al.
Truncating mutations on myofibrillar myopathies causing genes as prevalent molecular explanations on patients with dilated cardiomyopathy. Clin Genet 2017;92:616-23.

8. Ortiz-Genga MF, Cuenca S, Dal Ferro $\mathrm{M}$, et al. Truncating FLNC mutations are associated with high-risk dilated and arrhythmogenic cardiomyopathies. J Am Coll Cardiol 2016;68:2440-51.

9. Vorgerd M, van der Ven PF, Bruchertseifer $\mathrm{V}$, et al. A mutation in the dimerization domain of filamin c causes a novel type of autosomal dominant myofibrillar myopathy. Am J Hum Genet 2005;77:297-304.

10. Wang K, Li M, Hakonarson H. ANNOVAR: functional annotation of genetic variants from high-throughput sequencing data. Nucleic Acids Res 2010;38:e164.

11. Tavtigian SV, Deffenbaugh AM, Yin L, et al. Comprehensive statistical study of 452 BRCA1 missense substitutions with classification of eight recurrent substitutions as neutral. J Med Genet 2006;43:295-305.

12. Ng PC, Henikoff S. Predicting deleterious amino acid substitutions. Genome Res 2001;11:863-74.

13. Adzhubei IA, Schmidt S, Peshkin L, et al. A method and server for predicting damaging missense mutations. Nat Methods 2010;7:248-9.

14. Dong C, Wei P, Jian X, et al. Comparison and integration of deleteriousness prediction methods for nonsynonymous SNVs in whole exome sequencing studies. Hum Mol Genet 2015;24:2125-37.

15. Valdes-Mas R, Gutierrez-Fernandez A, Gomez J, et al. Mutations in filamin C cause a new form of familial hypertrophic cardiomyopathy. Nat Commun 2014;5:5326

16. Te Rijdt WP, van Tintelen JP, Vink A, et al. Phospholamban p.Arg14del cardiomyopathy is characterized by phospholamban aggregates, aggresomes, and autophagic degradation. Histopathology 2016;69:542-50.

17. Gomez J, Lorca R, Reguero JR, et al. Screening of the filamin $\mathrm{C}$ gene in a large cohort of hypertrophic cardiomyopathy patients. Circ Cardiovasc Genet 2017;10:2.

18. Brodehl A, Ferrier RA, Hamilton SJ, et al. Mutations in FLNC are associated with familial restrictive cardiomyopathy. Hum Mutat 2016;37:269-79.

19. Vermeer AM, van Engelen K, Postma $\mathrm{AV}$, et al. Ebstein anomaly associated with left ventricular noncompaction: an autosomal dominant condition that can 
be caused by mutations in MYH7. Am J Med Genet C Semin Med Genet 2013;163C:178-84.

20. Meredith C, Herrmann R, Parry C, et al. Mutations in the slow skeletal muscle fiber myosin heavy chain gene (MYH7) cause laing early-onset distal myopathy (MPD1). Am J Hum Genet
2004;75:703-8.

21. Tucker NR, McLellan MA, Hu D, et al. Novel Mutation in FLNC (Filamin C)

Causes Familial Restrictive Cardiomyopathy. Circ Cardiovasc Genet 2017;10:6.

22. Bhandary B, Meng Q, James J, et al. Cardiac fibrosis in proteotoxic cardiac disease is dependent upon myofibroblast TGF-beta signaling. J Am Heart Assoc 2018;7:e010013.

23. van Waning JI, Caliskan K, Hoedemaekers YM, et al. Genetics, clinical features, and long-term outcome of noncompaction cardiomyopathy. J Am Coll Cardiol 2018;71:711-22 